\title{
LASER ION SOURCE DEVELOPMENT FOR ISOL SYSTEMS AT RIA*
}

\author{
Y. Liü, C. Baktash, J. R. Beene, H. Z. Bilheux, C. C. Havener, H. F. Krause, \\ D. R. Schultz, D. W. Stracener, C. R. Vane, ORNL, Oak Ridge, TN 37831, U.S.A. \\ K. Wendt, Ch. Geppert, T. Kessler, K. Brueck, \\ Institut fuer Physik, Johannes Gutenberg-Universitaet Mainz, D-55099 Mainz, Germany
}

\begin{abstract}
A hot-cavity laser ion source based on solid-state tunable Ti:Sapphire lasers was tested. Three-photon resonant ionization schemes tested for $\mathrm{Sn}, \mathrm{Ge}$, and $\mathrm{Ni}$ yielded overall ionization efficiencies for the laser ion source of $22 \%, 3.3 \%$ and $2.7 \%$, respectively.
\end{abstract}

\section{INTRODUCTION}

Laser ion sources (LIS) based on resonant photoionization techniques are playing an increasingly vital role in improving the performance of existing isotope separator on-line (ISOL) radioactive ion beam facilities [1-4]. About 20 radioactive ion beams can now be routinely provided at CERN-ISOLDE using copper vapor laser-pumped dye lasers where source ionization efficiencies ranging from a few percent to nearly $30 \%$ have been realized [5]. Improvements in the ion source and laser technologies can, in principle, provide more efficient ionization with elemental selectivity of nearly $100 \%$ for about $80 \%$ of the elements in the periodic table. Improved LIS techniques will be needed at future advanced exotic beam accelerators such as the Rare Isotope Accelerator (RIA) [6].

Copper vapor laser (CVL) pumped dye laser systems have been the most widely used systems for LIS at existing ISOL facilities. However, these laser systems are costly to operate and maintain. Recently, tunable Ti:Sapphire lasers have emerged as an attractive all-solidstate alternative to the CVL-dye technology. Ti:Sapphire lasers are tunable over the $700-1000 \mathrm{~nm}$ range, and their range can be extended to the $200-500 \mathrm{~nm}$ region by optical harmonic generation. Despite their missing gap in the $500-700 \mathrm{~nm}$ range, Ti:Sapphire lasers are still very attractive due to their ruggedness, reliability, and small size.

The objective of this research effort is to study the feasibility of using Ti:Sapphire lasers as the principal laser system for LIS applications. A hot-cavity LIS system was constructed and tested at the off-line Ion Source Test Facility of the Holifield Radioactive Ion Beam Facility at the Oak Ridge National Laboratory. We describe the ion source and ionization schemes used for $\mathrm{Sn}, \mathrm{Ge}$, and $\mathrm{Ni}$, and report the measured ionization efficiency for these elements.

\footnotetext{
*Research sponsored by the U.S. Department of Energy, under contract DE-AC05-00OR22725 with UT-Battelle, LLC.

"liuy@ornl.gov
}

\section{HOT-CAVITY LASER ION SOURCE}

\section{Hot-cavity Ion Source}

A schematic view of the hot-cavity ion source assembly is shown in Fig. 1. Target materials are vaporized in the heated material reservoir. Atomic species of interest effuse through a transfer line into the tubular ionizer cavity where they are ionized by laser radiation entering from the right. The heated ionizer cavity is a $30 \mathrm{~mm}$ length Ta tube having a $3 \mathrm{~mm}$ inner diameter and a $1 \mathrm{~mm}$ wall thickness. The ions produced are extracted from the cavity and accelerated to an energy of $20 \mathrm{keV}$. The ionizer cavity and the transport tube are resistively heated to nearly $2000^{\circ} \mathrm{C}$. The direction of the DC electrical heating current flow also produces an axial electric field in the ionizer which pushes the ions towards the extractor. The target reservoir is heated radiatively to temperatures exceeding $1500^{\circ} \mathrm{C}$ using an independent cylindrical Ta heater.

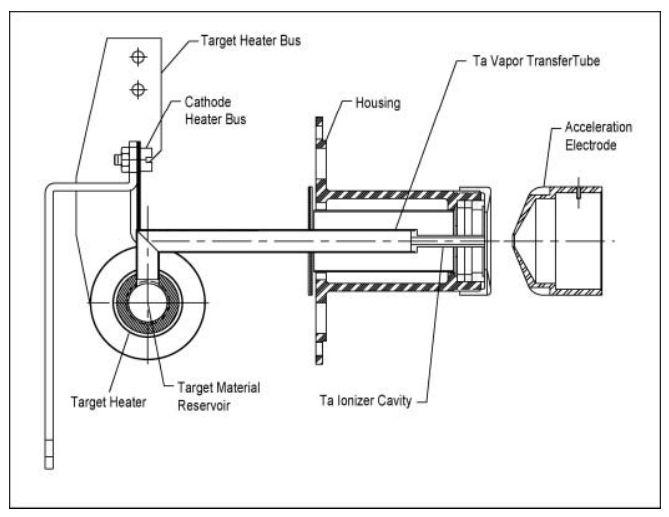

Fig. 1. Schematic drawing of the hot-cavity ion source with a Ta tubular ionizer ( $3 \mathrm{~mm}$ diameter).

\section{Ti:Sapphire Laser System}

The laser system, consisting of three Ti:Sa lasers pumped by a high repetition rate Q-switched Nd:YAG laser at $532 \mathrm{~nm}$, is shown schematically in Fig. 2. The Ti:Sapphire laser system was developed at the University of Mainz primarily for application in laser based ultratrace analysis and atomic spectroscopy studies [7]. The pump laser is a commercial frequency-doubled Nd:YAG laser obtained from Photonics Industries International, which provides $60 \mathrm{~W}$ average output power at $10 \mathrm{kHz}$ repetition rate. The Ti:Sapphire laser system generates narrowband $(\sim 3 \mathrm{GHz})$ tunable laser radiation in the 720 $925 \mathrm{~nm}$ (fundamental), 360-463 nm (frequency doubling) and 240-308 $\mathrm{nm}$ (frequency tripling) spectral regions. 
Temporal synchronization of the three laser pulses is better than $5 \mathrm{~ns}$ because the resonator in each laser is actively Q-switched using internal Pockels cells. All optical components required for beam monitoring, optimization and transport into the LIS were provided by the Mainz group for this study.

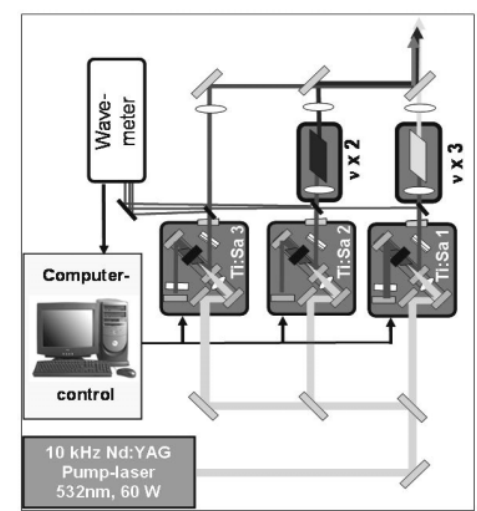

Fig. 2. Schematic view of the Ti:Sapphire laser system.

\section{RESULTS AND DISCUSSION}

Three-step resonant ionization of $\mathrm{Sn}, \mathrm{Ni}$ and Ge was studied. Sn was chosen as the primary benchmark species for efficiency measurements because $\mathrm{Sn}$ ionization schemes were established in studies using dye and Ti:Sapphire lasers at ISOLDE. Neutral atomic species of these elements were obtained by placing solid sample materials (tin metal foil, germanium metal shots, or nickel metal wires), into the heated target reservoir.

\section{Ionization Schemes}

To the best of our knowledge, no previous LIS studies have been reported for Ge. While Ni excitation schemes are known for the dye laser wavelength regime, the excitation ladders appropriate for Ti:Sapphire lasers had to be developed for this study. Our successful three-step resonant ionization schemes for $\mathrm{Sn}, \mathrm{Ge}$, and $\mathrm{Ni}$ are shown in Fig. 3. For all three elements, the first excitation step requires ultraviolet radiation obtained by frequency tripling of the fundamental laser beam, but the second and third steps use the fundamental output from the other two Ti:Sa lasers.

A known three-step excitation scheme (Fig. 3a) was used for Sn, which leads to an autoionization state in the last step [5]. The Sn atoms were first excited from the $5 \mathrm{p}^{2}$ ${ }^{3} \mathrm{P}_{0}$ ground state to the $5 \mathrm{p} 6 \mathrm{~s}{ }^{3} \mathrm{P}_{1}{ }^{0}$ state $\left(\lambda_{1}=286.42 \mathrm{~nm}\right)$, and then promoted with an infrared photon $\left(\lambda_{2}=811.62\right.$ $\mathrm{nm})$ to the $5 \mathrm{p} 6 \mathrm{p}^{3} \mathrm{P}_{2}$ state. Ionization was achieved in the last step leading to the $5 \mathrm{p} 9 \mathrm{~s}{ }^{3} \mathrm{P}_{2}{ }^{0}$ auto-ionization state at $59375.9 \mathrm{~cm}^{-1}$ by another infrared photon of $\lambda_{3}=823.67$ $\mathrm{nm}$. All three transitions were saturated and the saturation laser power densities were estimated to be $40 \mathrm{~mW} / \mathrm{cm}^{2}$ and $3 \mathrm{~W} / \mathrm{cm}^{2}$ for the first and second transition, respectively.

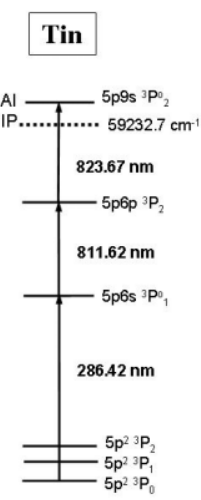

(a)

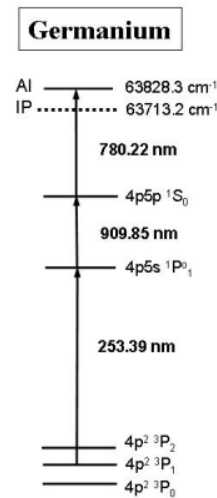

(b)

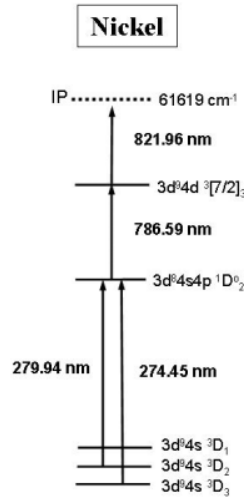

(c)
Fig. 3. Ionization schemes.

Resonant three-step ionization of Ge was obtained for the first time in this study. Assuming a pure Boltzmann distribution, the thermal populations of $\mathrm{Ge}$ in the hot cavity at $1700^{\circ} \mathrm{C}$ were $4 \mathrm{p}^{2}{ }^{3} \mathrm{P}_{0}(20.8 \%), 4 \mathrm{p}^{2}{ }^{3} \mathrm{P}_{1}(41.5 \%)$, and $4 p^{2}{ }^{3} \mathrm{P}_{2}$ (37.1\%). Thus, first excitation from the $4 \mathrm{p}^{2}$ ${ }^{3} \mathrm{P}_{1}$ level was selected (Fig. 3b). For the last transition, a search for auto-ionization or Rydberg states was conducted by scanning the third laser wavelength between $755 \mathrm{~nm}$ and $845 \mathrm{~nm}$. A rich spectrum of Rydberg states and several autoionization states were observed. The largest ionization yield was obtained using the autoionization state located at $63828.26 \mathrm{~cm}^{-1}$. Fig. 4 shows the measured $\mathrm{Ge}^{+}$current vs laser power in each excitation step. The data suggest that the last ionization step was not saturated.
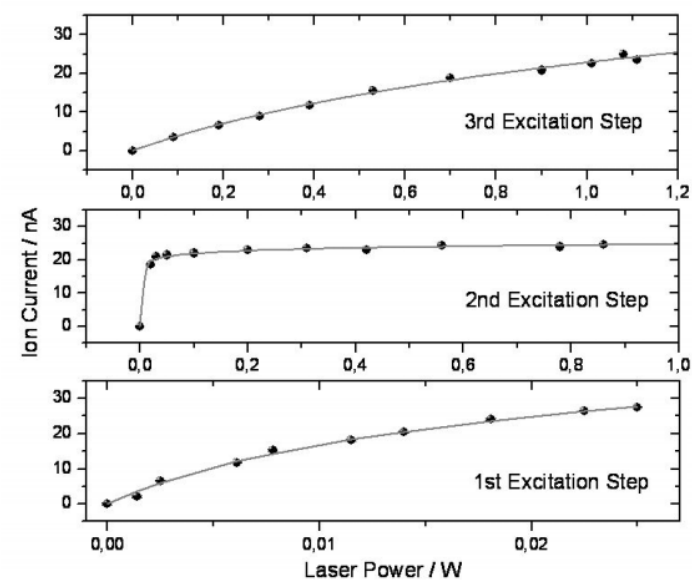

Fig. 4. Measured saturation curves for the three excitation steps in Ge.

For Ni, two different first excitation steps, starting from either the $3 \mathrm{~d}^{9} 4 \mathrm{~s}^{3} \mathrm{D}_{3}$ (population, $\mathrm{f},=26.7 \%$ ) or the $3 \mathrm{~d}^{9} 4 \mathrm{~s}$ ${ }^{3} \mathrm{D}_{2}$ (f $=11.66 \%$ ) level, and leading to the $3 \mathrm{~d}^{8} 4 \mathrm{~s} 4 \mathrm{p}{ }^{1} \mathrm{D}^{\mathrm{o}}{ }_{2}$ excited state, were used. As expected from the thermal populations, slightly higher $\mathrm{Ni}$ ion yield was obtained with the $3 \mathrm{~d}^{9} 4 \mathrm{~s}^{3} \mathrm{D}_{3} \rightarrow 3 \mathrm{~d}^{8} 4 \mathrm{~s} 4 \mathrm{p}{ }^{1} \mathrm{D}_{2}^{\mathrm{o}}$ transition. In the second excitation step, the $\mathrm{Ni}$ atoms were excited to the $3 \mathrm{~d}^{9} 4 \mathrm{~d}^{3}[7 / 2]_{3}$ state of even parity with an infrared photon $\left(\lambda_{2}=786.59 \mathrm{~nm}\right)$. The third laser wavelength was scanned over the laser output spectral range to search for auto- 
ionization states of odd parity. In the region near 62213.8 $\mathrm{cm}^{-1}$ and $62217.24 \mathrm{~cm}^{-1}$, two weak resonance peaks were detected with an enhancement of less than 3 over nonresonance ionization signals. A variety of Rydberg states located between 61070 and $61599 \mathrm{~cm}^{-1}$ or $0.068-0.003 \mathrm{eV}$ below the continuum were also detected. The excited $\mathrm{Ni}$ atoms in these Rydberg states were probably subsequently ionized via a collisional mechanism. The ionization yields via the Rydberg states were much larger than the above two weak resonances in the continuum. The strongest $\mathrm{Ni}$ ion signals were obtained from the Rydberg states near $61479.7 \mathrm{~cm}^{-1}$. The laser power available was about 10 $\mathrm{mW}$ for the first transition, and $1 \mathrm{~W}$ for the second and third steps, respectively. However, the first and the last transitions were not saturated.

The hot-cavity was operated in the temperature range of $1700-2000^{\circ} \mathrm{C}$. At these high temperatures, trace elements having low ionization potentials could also be ionized by surface ionization in the hot-cavity. No surface ionized ions of $\mathrm{Sn}, \mathrm{Ge}$, or Ni were observed, but strong signals of surface ionized trace elements $\mathrm{Cs}$ and Ga were measured, as shown in Fig. 5.
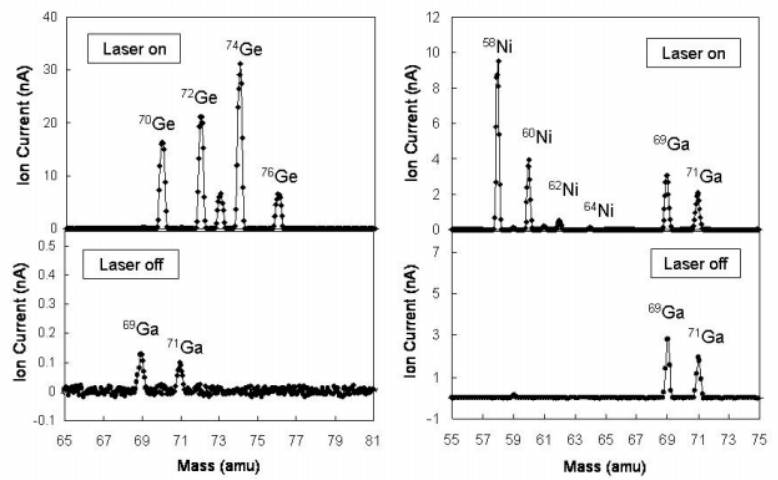

Fig. 5. Measured mass spectra of laser generated Ge and $\mathrm{Ni}$ ions (upper) and surface ionized $\mathrm{Ga}$ ions (lower).

\section{Ionization Efficiency}

The ionization efficiency for $\mathrm{Sn}, \mathrm{Ge}$ and $\mathrm{Ni}$ was measured using calibrated liquid samples, which contained feed stock material in the range of $10^{16}-10^{17}$ atoms per sample. During the efficiency measurements, the samples were heated to high temperatures, the laser wavelengths were tuned to the most efficient transitions investigated above, and the laser ion current was continuously recorded until the sample atoms completely evaporated out of the source. The ionization efficiency was calculated as the ratio of the integrated total number of detected ions to the total number of sample atoms contained in the initial sample.

Two efficiency measurements, conducted for Sn, yielded the values $21 \%$ and $23 \%$. Agreement of these results suggests that the operation of the laser system and the hot-cavity ion source was stable and reliable. The overall ionization efficiency of $\sim 22 \%$ for $\mathrm{Sn}$ obtained in this study is considerably higher than the $10 \%$ efficiency achieved using CVL pumped dye lasers and the 6.5\% efficiency obtained with Ti:Sapphire lasers at CERNISOLDE [5, 8]. This fact could be attributable to better laser-neutral overlap as well as better Sn vapor release in this work. A longer transport distance between the target reservoir and the ionizer cavity may also have contributed to the observed higher efficiency; ions generated by laser beams in the transfer line could be pushed towards the ionizer by the axial electric field.

The measured ionization efficiencies for $\mathrm{Ge}$ and $\mathrm{Ni}$ were $3.3 \%$ and $2.7 \%$, respectively. The $2.7 \%$ efficiency for $\mathrm{Ni}$ is less than the $>6 \%$ efficiency obtained with CVLdye lasers [5]. One of the reasons for the low ionization efficiency is the fact that the first and the third excitation steps are not saturated in this work. Other data also suggest that atomic Ni may react with the hot transport tube material $(\mathrm{Ta})$, potentially undergoing chemical, eutectic, or alloy trapping. As a result, the overall ionization efficiency is lowered.

Development of more efficient excitation and ionization schemes for $\mathrm{Ni}$ and $\mathrm{Ge}$ are in progress. Systematic spectroscopic studies on the auto-ionization and Rydberg states employed in the last ionization step will be conducted in the near future. Improvements in mechanical design and material properties of the hot-cavity ion source will be studied in order to improve the release and transport of atomic species towards the laser ionization region and reduce surface ionized ions.

\section{REFERENCES}

[1] Kluge H.-J. , Ames F., Ruster W., Wallmeroth K., Proc. Acc. Radioact. Beams WS, TRI 85 (1) (1985) 119-129.

[2] V.N. Fedoseyev, et al., Nucl. Instr. and Meth. B 204 (2003) 353-358.

[3] Yu. Kudryavtsev, et al., Nucl. Phys. A 701 (2002) $465 c-469 c$.

[4] A.E. Barzakh, V.P. Denisov, D.V. Fedorov, S.Yu. Orlov, M.D. Seliverstov, Nucl. Instr. and Meth. B126 (1997) 85.

[5] U. Koester, V.N. Fedoseyev, V.I. Mishin, Spectro. Acta Part B58 (2003) 1047.

[6] http://www.orau.org/ria/ria-whitepaper-2000.pdf.

[7] C. Grüning, G. Huber, P. Klopp, J.V. Kratz, P. Kunz, G. Passler, N. Trautmann, A. Waldek, K. Wendt, Int. J. Mass Spec. 235, 171-178 (2004).

[8] K. Brück, W. Eller, Ch. Geppert, T. Kessler, J. Lettry, M. Lindroos, F. Wenander, K. Wies, R. Wilfinger, K. Wendt, Rev. Sci. Instr., to be published. 